\title{
An Explanation of Fitts' Law-like Performance in Gaze-Based Selection Tasks Using a Psychophysics Approach
}

\author{
Immo Schuetz \\ Facebook Reality Labs \\ Redmond, Washington \\ ischtz@fb.com \\ Kevin J. MacKenzie \\ Facebook Reality Labs \\ Redmond, Washington \\ kjmacken@fb.com
}

\author{
T. Scott Murdison \\ Facebook Reality Labs \\ Redmond, Washington \\ smurdison@fb.com \\ Marina Zannoli \\ Facebook Reality Labs \\ Redmond, Washington \\ marinazannoli@fb.com
}

\begin{abstract}
Eye gaze as an input method has been studied since the 1990s, to varied results: some studies found gaze to be more efficient than traditional input methods like a mouse, others far behind. Comparisons are often backed up by Fitts' Law without explicitly acknowledging the ballistic nature of saccadic eye movements. Using a vision science-inspired model, we here show that a Fitts'-like distribution of movement times can arise due to the execution of secondary saccades, especially when targets are small. Study participants selected circular targets using gaze. Seven different target sizes and two saccade distances were used. We then determined performance across target sizes for different sampling windows ("dwell times") and predicted an optimal dwell time range. Best performance was achieved for large targets reachable by a single saccade. Our findings highlight that Fitts' Law, while a suitable approximation in some cases, is an incomplete description of gaze interaction dynamics.
\end{abstract}

\section{CCS CONCEPTS}

- Human-centered computing $\rightarrow$ Pointing devices; Pointing; User models; User studies.

\section{KEYWORDS}

Fitts' Law; user interfaces; gaze input; eye movements; gaze selection; saccades

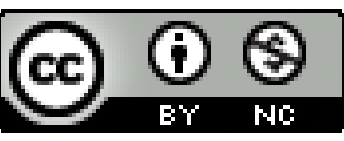

This work is licensed under a Creative Commons AttributionNonCommercial International 4.0 License.

(C) 2019 Copyright held by the owner/author(s).

ACM ISBN 978-1-4503-5970-2/19/05.

https://doi.org/10.1145/3290605.3300765
ACM Reference Format:

Immo Schuetz, T. Scott Murdison, Kevin J. MacKenzie, and Marina Zannoli. 2019. An Explanation of Fitts' Law-like Performance in Gaze-Based Selection Tasks Using a Psychophysics Approach. In CHI Conference on Human Factors in Computing Systems Proceedings (CHI 2019), May 4-9, 2019, Glasgow, Scotland UK. ACM, New York, NY, USA, 13 pages. https://doi.org/10.1145/3290605.3300765

\section{INTRODUCTION}

Using eye gaze to interact with technology is a promising idea: tracking gaze position can potentially allow for fast, intuitive and hands-free interaction. As our point of gaze often represents the focus of visual attention, such a system could also provide more context-aware interaction than using traditional input devices [40]. Gaze-based interaction techniques have been studied since the early 1990s [29, 56], and assistive technologies based on eye tracking are in practical use today [39]. The inclusion of eye trackers in emerging technologies such as Virtual and Augmented Reality (VR/AR) further drive a renewed interest in gaze-based interaction schemes (e.g., [6, 22, 39, 46]). In the context of selection, for example when picking one of multiple available menu items using a mouse pointer, Fitts' Law [16] has emerged as a gold standard to evaluate and compare the efficiency of different Human-Computer Interaction (HCI) paradigms [37]. Originally rooted in information theory [16], later accounts describe it as a good model of target-directed movements under continuous control $[11,42]$. In the mouse pointer example, this means that the user continuously adjusts movement direction using visual feedback of the on-screen pointer until they reach the target in order to compensate for movement error.

Because looking at a target to make a selection is reminiscent of a pointing task, Fitts' Law seems like an obvious way to compare gaze selection to other input modalities. 


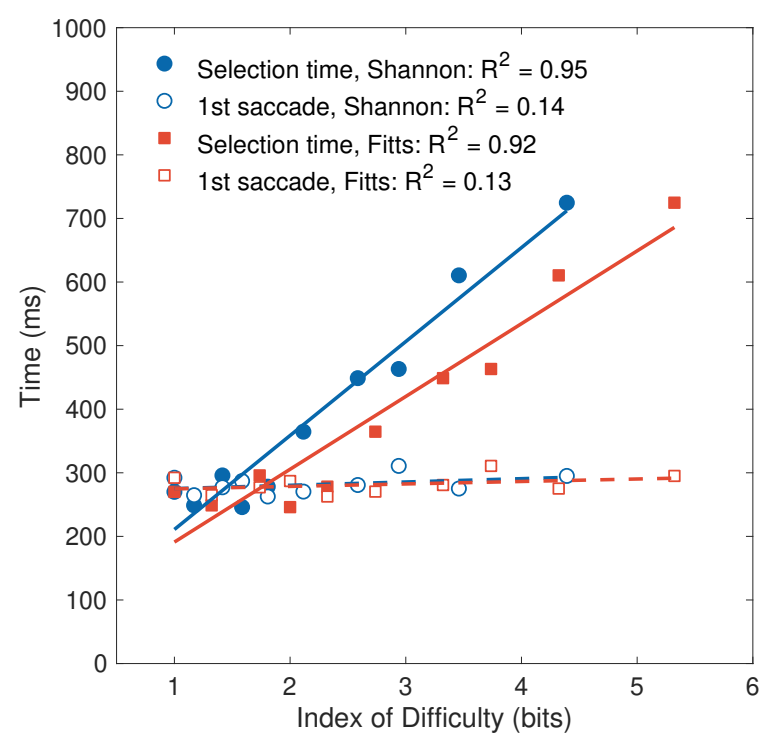

Figure 1: Target selection times (filled markers, solid lines) and first saccade end times (open markers, dashed lines) in every trial, averaged as a function of Index of Difficulty (ID) as calculated using Fitts' formulation (red squares; see Results section for details) and Shannon formulation (blue circles). Selection time includes all eye movements until gaze falls within the target for $50 \mathrm{~ms}$ and shows a pattern akin to Fitts' Law, while first saccades yield movement times that are independent of ID (see Table 2 for intercept, slope and model fit parameters).

Indeed, gaze selection has been compared to many other interaction paradigms using Fitts' Law, such as touch, mouse, and head pointing (e.g., [22, 43, 46, 51, 56, 61]). However, eye movements are fundamentally different from visually controlled hand or mouse movements, warranting a more detailed look at whether Fitts' Law can accurately describe gaze-based selection. Our eye movements follow a pattern of alternating periods of fixation, when eye positions are relatively stable and visual information is sampled from the environment, and fast eye movements called saccades, that can reach angular velocities of up to $900 \%$ s ([1], see e.g. [32] for a detailed review). Most importantly, peak velocity of saccades, and by extension movement duration, are strictly defined by movement amplitude and independent of the saccade target size $[1,9,23,58]$. At the same time, the oculomotor system is mostly unable to utilize visual feedback to adjust eye movements in an 'online' manner as we do with pointing movements. Therefore, saccades are pre-programmed, ballistic movements [50, 54]. Given these reasons, Fitts' Law should not apply to saccadic eye movements. Put differently, using a mouse in the same way would mean giving the mouse a single poke and hoping for the cursor to land close to the target.
How, then, can these oculomotor findings be reconciled with the large body of studies that report good correlations between movement time and Index of Difficulty (ID) for gaze selection tasks? As we will show below, movement times of individual saccades are indeed independent of target size and do not follow Fitts' Law. However, humans frequently generate secondary ("corrective") saccades after a main targetdirected saccade, especially when aiming for small targets $[4,33,45,60]$. We show that these additional saccades can explain the Fitts-like relationship between movement time and target size. This phenomenon can still serve as a valuable tool for the evaluation of gaze-based input paradigms, at least for small targets that elicit secondary saccadic eye movements. However, we will point out below that the underlying biomechanics for pointing and gaze selection are fundamentally different, and gaze interaction designers should therefore be careful when drawing theoretical conclusions on gaze from established Fitts' Law paradigms.

\section{KEY CONTRIBUTIONS}

(1) We reconcile current $\mathrm{HCI}$ and oculomotor literature and show that corrective saccades performed after the first (main) saccade explain a Fitts-like target size dependency in gaze selection without drawing into question the ballistic nature of individual saccades.

(2) Using an approach inspired by Psychophysics, we describe a new way of quantifying performance in an eye gaze selection task for different target sizes and dwell times.

(3) We suggest optimal target sizes for best selection performance, as well as quantify when the described Fittslike relationship can predict selection time, informing future interaction design.

\section{RELATED WORK AND BACKGROUND}

\section{Eye Gaze for Selection}

The first systematic investigation of eye gaze as an input modality was performed 1987 by Ware and Mikaelian [56], who found gaze input to be potentially much faster than a mouse, especially when paired with a hardware button to confirm selection. Since then, work in this field has focused mainly on how gaze input compares to more traditional input methods, such as a mouse, button, or touch input, and has yielded conflicting results. Sibert and Jacob (2000) report faster selection using gaze than the mouse [51], and Tanriverdi and Jacob later found a similar speed advantage for the eyes compared to a 3D motion-tracked pointer in a VR setup [53]. Other investigations found longer selection times and lower performance for gaze compared to the mouse $[43,61]$ or comparable performance [49]. 

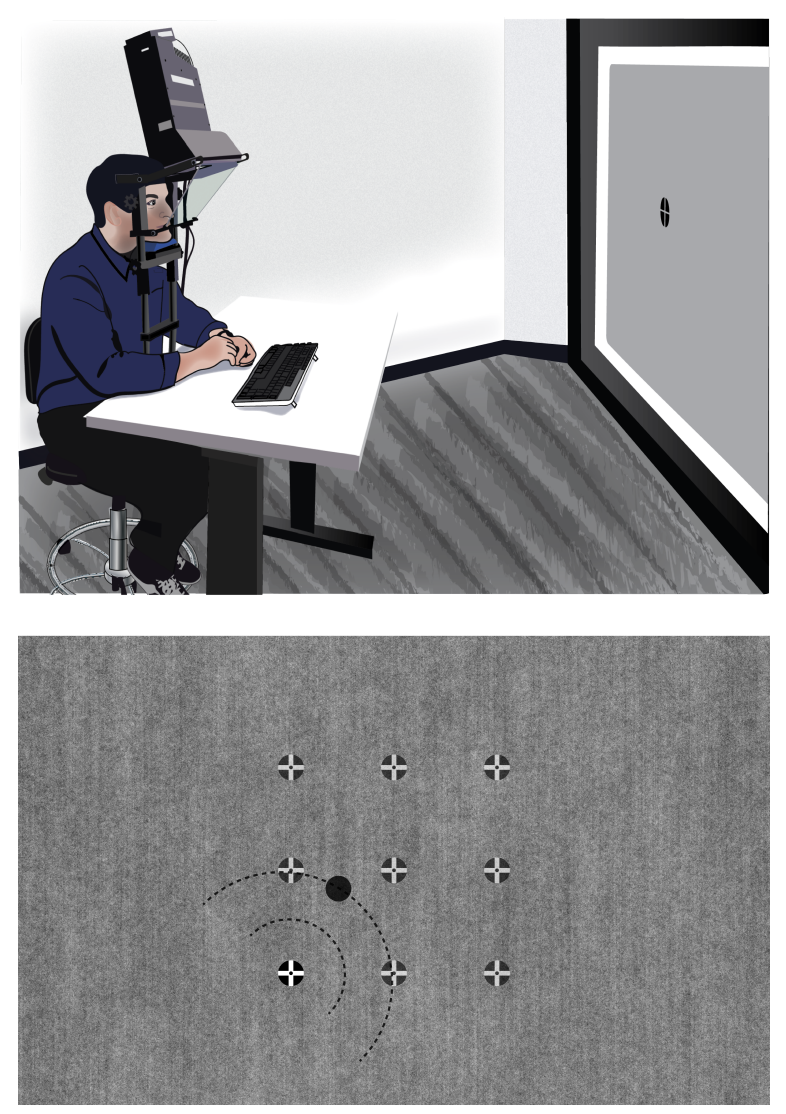

\begin{tabular}{c|c|c} 
fixation & target & ISI \\
\hline $500-1250 \mathrm{~ms}$ & $2000 \mathrm{~ms}$ & $1250-2000 \mathrm{~ms}$
\end{tabular}

Figure 2: Experimental Setup and Task. Top: Visual stimuli were presented on a back-projection screen at $2560 \times 1600$ pixels, while the participant's eye movements were tracked using an Eyelink 1000plus tracker integrated into a chin and forehead rest. Illustration courtesy of Benjamin Facer. Bottom: Potential starting locations (fixation crosses, not to scale) and one example target used during the experiment. Targets (black circle) were presented on a semicircle of 5 or $10{ }^{\circ}$ radius around the starting location at a randomly chosen angle (dotted lines; displayed for one starting location only). The provided timeline illustrates trial timing.

Recent technological advances have sparked growing interest in gaze-based HCI. First, the advent of inexpensive and comparatively accurate eye tracking devices has brought gaze interaction from the medical and assistive domains into everyday computing [40], and second, new immersive technologies such as VR and AR are already starting to incorporate eye tracking technology (e.g., [6, 46]), allowing for as-yet unimagined interaction paradigms. Two recent studies applied ISO 9241-9 (an industry standard for the evaluation of input devices, [17]) to gaze pointing using the FOVE $0^{1}$ headmounted display (HMD), with inconclusive results: gaze was either faster than head- but slower than mouse pointing [22], or found to be even slower than head pointing [46]. Another study compared gaze and head pointing for different simulated AR fields of view (FOV) using a different eye tracker and found a clear advantage for gaze pointing especially at larger FOV [6]. It is likely that at least some of these discrepancies can be explained by differences in tracking fidelity.

While many existing studies report their achieved gaze precision and accuracy values, not much work has explicitly investigated the relationship between eye tracker fidelity and selection performance for interface elements of varying size. Poor eye tracking quality should manifest in higher error rates especially for smaller targets, but not necessarily in longer target selection times, especially if only correct selections are analyzed. Earlier work found increased selection error rates for target sizes below 1.5-2 degrees [20, 56], while recent studies using a VR HMD report a minimal target size of about $3^{\circ}$ and mention difficulties in calibrating the eye tracker $[22,46]$. The same group recently reported an average calibration accuracy of $3.9-4.34^{\circ}$ for this eye tracker (FOVE; [47]). The range of these values highlight the necessity of good eye tracking fidelity to achieve robust target selection, and newer approaches are already circumventing this limitation by adapting user interfaces (UIs) to the available gaze tracking performance, for example by dynamically changing the size of UI elements $[2,14]$.

When we use our eyes to explore a visual display such as a computer monitor, simply triggering actions based on the current gaze point would lead to selection of every glanced-at element of the display. To overcome this problem, termed the "Midas Touch problem" [29], the "pointing" action (choosing an element) must be separated from the "selection" action (triggering the action linked to the chosen element). One way to accomplish this using only eye movements is to include a "dwell time", i.e., a period of time that the (gaze-) pointer needs to "dwell" on the target to execute a selection [21, 41]. Typical reported dwell times range from 150 [51,52] or 200 $\mathrm{ms}$ [43] up to $750 \mathrm{~ms}$ [61]. As we will argue below, this range of dwell times is in good agreement with the time course of human eye movements during a target selection task.

\section{Does Fitts' Law apply to eye gaze?}

First published in 1954, Fitts' Law has become a gold-standard measure of interaction efficiency in $\mathrm{HCI}$ [37]. The law relates Movement Time (MT) in a target-directed movement task to the ratio of movement amplitude and the size of the target, expressed as an Index of Difficulty (ID) [16]. Thus, Fitts' law

\footnotetext{
${ }^{1}$ https://www.getfove.com/
} 
captures the inherent speed-accuracy trade-off when moving towards a target of limited size, and the framework has been shown to hold in a variety of motor contexts such as arm [18], wrist [11], and head movements [30]. Fitts' original model was rooted in information theory, likening the distance of a movement to the "signal" and target width to the "noise" in an information transfer scenario [16]. Later models explained the resulting relationship as an ongoing motor control process in which visual feedback is incorporated at each stage of the movement to compensate for motor errors. For example, the widely-cited model of Crossman and Goodeve assumed a sequence of discrete "submovements" that reduce the remaining distance to the target on each step by a proportional amount, leading to a movement time distribution in agreement with Fitts' Law $[11,59]$. In another model by Meyer et al., Fitts' Law is derived from the trade-off between the duration of a primary submovement and the probability of executing an optional second submovement to achieve motor error correction [42].

While models based on visual feedback work well for motor tasks such as reaching and pointing, the extreme velocities of saccadic eye movements (up to $900 \%$ [1]) and resulting blur of the retinal image prevent the oculomotor system from utilizing on-line visual feedback [7]. In fact, visual input is mostly suppressed while the eye is in motion $[8,48]$. In contrast to e.g. arm movements, saccades are therefore ballistic movements that are programmed and executed based on an internal control model $[10,50,54]$. This leads to a characteristic relationship between saccadic amplitude and peak velocity that has been termed the saccadic "main sequence" $[1,23,58]$; by extension, this means that saccade duration or movement time is completely determined by amplitude independent of the size of the saccade target [9]. Yet, published user studies found good correspondence between movement times and ID that goes beyond a simple relation to movement amplitude. How does Fitts' Law relate to ballistic saccadic control?

The discrepancy between Fitts' Law as a description of movements under continuous control and the ballistic nature of saccades has been raised before $[12,13]$, and early reports were somewhat divided on the applicability of Fitts' Law to gaze. The first published account by Ware and Mikaelian (1987) found good correlations between MT and ID, but the authors were careful to emphasize that they had used Fitts' Law only as "a convenient way of summarizing the results", not "to make any theoretical claims" [56]. Sibert and Jacob analyzed their findings using Welford's formulation of Fitts' Law [57] and found that the resulting regression slope was low and movement times were overall better predicted purely by saccade amplitude $[51,52]$, whereas Miniotas (2000) reported a good model fit as evidenced by values of $R^{2}>0.99$ (but concluded that the mouse was faster in selecting a target ribbon than gaze [43]).

The apparent discrepancies between oculomotor control literature and the above findings on gaze selection times resembling Fitts' Law could be resolved by considering the act of selecting a target using gaze not as the product of a single saccade, which would indeed be under ballistic control, but as a sequence of a main and one or more secondary ("corrective") saccades. Target-directed saccades show errors of around $5-10 \%$, especially when aiming for small, pointlike targets [33], and additional, brief saccades are often dispatched after this main saccade to bring the gaze point closer to the intended target [3, 4, 45]. Wu, Kwon and Kowler (2010) found that movement times for sequences of saccades to multiple targets followed Fitts' Law [60]. The increase of total movement time with ID was associated with an increasing frequency of secondary saccades at larger IDs, while latency and duration of main saccades were unchanged and only depended on movement amplitude. Even though their account was not directly an investigation into gaze as an interaction technique, this finding parallels the submovement-based models for pointing movements [11, 42, 59]. Indeed, theoretical work suggests that as few as two such submovements are necessary to achieve a relationship between movement time and ID that resembles Fitts' Law [26, 34, 35]. Hoffmann (2016) further suggested that the likelihood of requiring additional submovements to capture a target explains most of the relationship between movement time and ID and thus the resulting Fitts-like pattern [26]. Together, these results imply that a higher likelihood of corrective saccades for targets with high ID could explain the above findings on Fitts' Law in gaze interaction. Because humans rarely execute more than one corrective saccade [60], the underlying motor control strategy is quite different from the continuous feedback control assumption that lies at the heart of Fitts' Law. Rather, gaze selection times should follow the "Fitts-like" relationship discussed in [26], which leads to an approximation of the true Fitts' Law with two submovements. While this relationship can still be a helpful predictor of selection times in gaze interaction, it should further only be applicable in cases where secondary saccades actually occur (i.e., small targets with high ID).

Investigations on Fitts' Law for pointing movements typically find an inflection point in the relationship between movement time and ID where MT is approximately constant below a certain value of ID (e.g., [11, 16]). Gan and Hoffmann (2007) argue that these cases lead to very short movement times (under $200 \mathrm{~ms}$ ) for which visual feedback would not improve accuracy, and where movements are consequently executed ballistically [18]. A recent literature review found values between 1.5 and 4 for this critical Index of Difficulty $\left(I D_{\text {crit }}\right)$ in different effector movements (e.g., hand, 
leg, or body movements, depending on the muscle forces and masses involved in the movement) [25]. In the data of Wu et al., saccadic sequences appear to agree with Fitts' Law down to their smallest chosen value of ID $=1.5$, accompanied by an increase in secondary saccades $[25,60]$. It follows that, if secondary saccades do explain the ID-MT relationship, there should be a critical target size (and corresponding ID) above which secondary saccades become unlikely and this relationship disappears - an $I D_{\text {crit }}$ for gaze interaction.

In summary, treating gaze as a complex, adaptive movement sequence leads to the following clear predictions on the relation between selection time and target difficulty in gaze interaction: Below a critical ID and above the corresponding target size, only a single saccade should be necessary to reach the target and movement time should be purely predicted by saccade amplitude [9]. Above $I D_{\text {crit }}$, secondary saccades are likely to occur and this should lead to an increase in MT with ID akin to Fitts' Law [60]. Finally, eye tracking system fidelity should limit the range of possible target sizes at the lower end, since targets below a minimum size should not be selectable due to inaccuracies in gaze tracking even under optimal user behavior.

\section{USER STUDY}

To investigate the relationship between saccade behavior and target selection performance, we designed a user study in which participants selected targets of different sizes using their gaze.

\section{Participants}

Sixteen volunteers participated in our user study (10 male, 6 female; mean age $27.8 \pm 5.7$ years). All participants had normal or corrected-to-normal vision, no known history of visual or oculomotor deficits and provided written informed consent before taking part in the study. Participants were recruited via social media, email and word of mouth, and gift cards were provided as a gratuity for participation.

\section{Experimental Setup}

Participants sat in front of a projection screen at a distance of $1.8 \mathrm{~m}(243 \times 152 \mathrm{~cm}$; Da-Lite, Inc, Warsaw, IN, USA), with the midpoint between their eyes aligned with the center of the screen and their head stabilized using a chin and forehead rest (Fig. 2, top). The projected image spanned a field of view $(\mathrm{FOV})$ of $73 \times 46$ degrees at a resolution of $2560 \times$ 1600 pixels (X-Vision WQ LED INF-R; Digital Projection, Inc, Atlanta, GA, USA). All visual stimuli except for eye tracker calibration targets were presented superimposed on pink noise images, designed to be uniform in contrast energy while mimicking the statistics of natural visual scenes [15]. Eye movements were recorded using an Eyelink 1000 plus (SR Research, Ottawa, ON, Canada) video-based eye tracker at sampling rate of $1000 \mathrm{~Hz}$. At the start of the experiment and before each experimental block, the eye tracker was calibrated using the built-in 13-point calibration procedure. Calibration accuracies as reported by the system ranged from $0.25-1.30^{\circ}$ (mean $0.50^{\circ}$, median $0.47^{\circ}$ ).

\section{Task and Experimental Design}

In each trial, participants made eye movements from a starting location to a circular target. Starting locations were presented pseudo-randomly in one of nine positions (screen center or $\pm 10^{\circ}$ horizontally and/or vertically; cf. Fig. 2, bottom) and were indicated by a fixation cross. Participants fixated the cross and pressed the space bar to start each trial, after which gaze position was recorded for $250 \mathrm{~ms}$ to establish a trial-by-trial baseline of gaze accuracy and precision. Following a randomized delay of 250-1000 ms, a circular target was presented at a $5^{\circ}$ or $10^{\circ}$ distance from the starting location, at a random angle on a semicircle oriented towards the center of the screen. We followed this procedure to avoid adaptation to a specific saccade direction and amplitude, which can influence saccade behavior [55]. Targets were presented with seven different target sizes (diameter of target circle: $0.5,1,1.5,2,3,4$, and $5^{\circ}$ ) in randomized order. Gaze position was recorded for $2000 \mathrm{~ms}$ after target onset, after which the target disappeared and a blank pink noise background was presented for a randomized inter-trial interval (ITI) of 1250$2000 \mathrm{~ms}$ before the start of the next trial, as depicted in the timeline in Fig. 2 (bottom).

Trials were presented in blocks of 28 (7 target sizes $\times 2$ saccade amplitudes $\times 2$ repetitions). After each block, participants had the option to take a break, and the eye tracker was re-calibrated before the start of the next block. Starting locations were randomized across each block.

\section{Gaze Data Preprocessing}

All eye movement analyses were performed using MATLAB (The MathWorks, Inc., Natick, MA, USA). Gaze data were imported from raw Eyelink data files and split into individual trial time series. We classified saccades during the $2 \mathrm{~s}$ target presentation interval using the manufacturer-provided algorithm at default thresholds for saccade velocity and acceleration $\left(30^{\circ} / \mathrm{s}\right.$ and $8000^{\circ} / \mathrm{s}^{2}$, respectively). Saccades with durations outside a range of $20-100 \mathrm{~ms}$ were discarded as artifacts. To analyze saccade end times, we extracted gaze positions in the screen plane for detected offset times of the first (up to) three saccades in each trial. Additionally, we calculated the median gaze position for a range of sampling windows between 0 and $1500 \mathrm{~ms}$ after the end of the first (main) saccade in steps of $10 \mathrm{~ms}$. Thus, whenever we refer to the sampling window in the following sections, times are specified relative to the end of the first saccade. 




Figure 3: Selection time (time between target onset and gaze entry into the target circle; black lines) and saccade end times for the first two saccades per trial (solid colored lines) as well as third saccades when detected in more than $2.5 \%$ of trials (dotted line), plotted as a function of target size. Error bars show +/- $1 \mathrm{SE}$.

\section{RESULTS AND ANALYSES}

\section{Selection Time}

Our gaze selection task did not include an explicit mode of selection, such as a button press to indicate that the participant was sure their gaze was resting on the target (cf. [22]). Consequently, a definition of selection time is necessary before we can compare such times to those predicted by Fitts' Law. We measured selection time as the time between target onset and when gaze first entered the target. To account for eye tracker variability, the latter time point was defined as the first sample of a $50 \mathrm{~ms}$ sliding window in which all gaze samples lay completely within the target circle. Effectively, this yielded the time until the first period of stable gaze on the target, independently of any detected eye movement events during this time frame.

Resulting selection times for each presented target size are plotted as the black line in Fig. 3. Colored lines in the same figure represent average saccade end times relative to target onset for the first, second, and third saccade occurring in each trial. Selection times varied with target size, while individual saccade end times did not. To quantify the individual contributions of distance and target size on selection timing, we performed a 2 (distance) $\times 7$ (target size) RMANOVA on selection times and first and second saccade end times. Since tertiary saccades were generally infrequent and only occurred for target sizes below $2^{\circ}$, we did not perform
RM-ANOVAs for third saccades. Average end times of third saccades are provided in Fig. 3 for reference only, drawn for conditions where at least $2.5 \%$ of trials included three or more saccades. ANOVA results for selection time and saccade end times are presented in Table 1. Smaller targets led to longer selection times (median MT: $738 \mathrm{~ms}$ at $0.5^{\circ}$ to $232 \mathrm{~ms}$ at $5^{\circ}$ ), but first and second saccade end times were independent of target size (median MT across all trials: $232 \mathrm{~ms}$ and $425 \mathrm{~ms}$, see Fig. 3). Saccade distance ( 5 or $10^{\circ}$ ) had no effect on all measured times and no interaction emerged.

\section{Gaze Selection and Fitts' Law}

Results presented in Table 2 suggest that selection time was strongly influenced by target size, while first and second saccade end times were independent of target parameters. To investigate whether Fitts' Law, as commonly used in pointing movements, predicts differences in selection time, we next calculated Indices of Difficulty (ID) for all conditions and performed a linear regression analysis on selection and saccade end times with ID. Fitts' Law relates movement time (MT) to ID using a linear model of the form:

$$
M T=a+b I D
$$

ID is generally defined as a function of movement amplitude (A) and target width (W), but different formulations to derive ID from $\mathrm{A}$ and $\mathrm{W}$ have been proposed over the years (see Introduction section). To highlight that a Fitts' Law-like pattern of movement times can emerge in gaze selection regardless of the specific formulation used, we compared our data to Fitts' original formulation $\left(I D_{F} ;[16]\right)$ :

$$
I D_{F}=\log _{2}(2 A / W)
$$

as well as the Shannon formulation $\left(I D_{S} ;[36,38]\right)$ used in the ISO 9421-9 standard [17]:

$$
I D_{S}=\log _{2}(A / W+1)
$$

Combining our chosen values for distance and target size according to these formulations led to 10 individual values for ID in the range of $1.0-4.4$ bits $\left(I D_{S}\right)$ and $1.0-5.3$ bits $\left(I D_{F}\right)$. Table 2 includes linear regression parameters for selection and saccade end times, as well as $R^{2}$ and F-test results to determine goodness of fit. Results for selection and first saccade end times are also visualized in Fig. 1 for comparison. Selection times showed a strong linear relationship with ID for both formulations, similar to that predicted by Fitts' Law for these IDs (Fitts: $R_{F}^{2}=0.92, \mathrm{~F}=89.6$; Shannon: $R_{S}^{2}=0.95$, $\mathrm{F}=151.8$ ), while end times of the first saccade were not predicted by ID and in fact displayed slopes close to zero, meaning saccade end latencies were approximately constant. End times of the second saccade were predicted by ID with a reasonable linear model fit $\left(R_{F}^{2}=0.79, \mathrm{~F}=31.0 ; R_{S}^{2}=0.79, \mathrm{~F}\right.$ 
Table 1: RM-ANOVA results for effects of saccade amplitude and target size on selection times as well as first and second saccade end times. Values in italics were corrected using Greenhouse-Geisser correction for violations of sphericity. ${ }^{1} \mathrm{~N}=14$ because two participants did not perform more than one saccade in the largest $\operatorname{target}$ size $\left(5^{\circ}\right)$ condition.

\begin{tabular}{llccccccccc}
\hline Time & $\mathbf{N}$ & \multicolumn{3}{c}{ Distance } & \multicolumn{3}{c}{ Target Size } & \multicolumn{3}{c}{ Interaction } \\
& & $F$ & $p$ & $\eta_{G}^{2}$ & $F$ & $p$ & $\eta_{G}^{2}$ & $F$ & $p$ & $\eta_{G}^{2}$ \\
\hline 1st Saccade & 16 & 3.4 & 0.087 & 0.01 & 0.6 & 0.578 & 0.01 & 0.9 & 0.495 & 0.01 \\
2nd Saccade & $14^{1}$ & 2.6 & 0.131 & 0.01 & 0.6 & 0.569 & 0.02 & 0.9 & 0.478 & 0.02 \\
Selection Time & 16 & 0.8 & 0.384 & 0.00 & 57.1 & $<0.001$ & 0.57 & 0.6 & 0.631 & 0.01 \\
\hline
\end{tabular}

Table 2: Model parameters for Fitts' Law applied to movement and saccade end times for Fitts' formulation $\left(I D_{F}\right)$ and the Shannon formulation of ID $\left(I D_{S}\right)$. See also Fig. 1.

\begin{tabular}{llrrcrl}
\hline ID & Time & $\mathbf{a}$ & $\mathbf{b}$ & $\mathbf{R}^{2}$ & $\mathbf{F}$ & $\mathbf{p}$ \\
\hline $\mathrm{ID}_{F}$ & 1st & 270.84 & 3.88 & 0.13 & 1.2 & 0.307 \\
& 2nd & 784.82 & -51.74 & 0.79 & 31.0 & $\mathbf{< 0 . 0 0 1}$ \\
& Sel & 76.58 & 114.48 & 0.92 & 89.6 & $\mathbf{< 0 . 0 0 1}$ \\
\hline $\mathrm{ID}_{S}$ & 1st & 269.94 & 5.20 & 0.14 & 1.4 & 0.278 \\
& 2nd & 787.91 & -65.45 & 0.79 & 30.1 & $\mathbf{< 0 . 0 0 1}$ \\
& Sel & 63.17 & 147.74 & 0.95 & 151.8 & $\mathbf{< 0 . 0 0 1}$ \\
\hline
\end{tabular}

$=30.1$ ), but with a negative slope coefficient. In other words, for low-ID ("easier") targets, second saccades on average ended later than for high-ID ("difficult") targets.

\section{Critical Index of Difficulty}

Similar to other reports $[18,25]$, selection times in Fig. 1 appear to transition to a horizontal slope between IDs of 1 and approximately 2 . This pattern has recently been explained as a switch from visually-controlled to ballistic movements below a critical ID (ID crit, [25]). To determine the value of $I D_{\text {crit }}$, we repeated the above regression analysis whilst leaving out the smallest, then two smallest, etc., ID values. We defined $I D_{\text {crit }}$ as the largest excluded ID value that maximized regression fit for the remaining IDs. To account for the resulting differences in sample size and compare model fit to that achieved using the full range of IDs (shown in Fig. 1 ), adjusted $R^{2}\left(R_{A}^{2}\right)$ was used to determine goodness of fit.

The best agreement with the Shannon formulation of ID was achieved for $I D_{\text {crit }}=1.42\left(R_{A}^{2}=0.980\right)$ and model fit for the reduced ID range was better than for the full range of IDs $\left(R_{A}^{2}=0.944\right)$. Using Fitts' formulation, we found similar values at $I D_{\text {crit }}=1.74$, and limiting the range of IDs to those above this value again improved model fit $\left(R_{A}^{2}=\right.$ $0.984)$ compared to the full range $\left(R_{A}^{2}=0.908\right)$. Due to our chosen combinations of saccade amplitude and target size, both values correspond to the same condition $\left(3^{\circ}\right.$ target at $5^{\circ}$ amplitude), suggesting that for targets larger than $3^{\circ}$, only a single saccade was necessary for selection and the ID-MT relationship was reduced to a constant reaction time.

\section{Increase in Secondary Saccades with ID}

The theoretical modeling approach described by Hoffmann [26] suggested that a Fitts-like relationship between movement time and ID can emerge with as few as two submovements, and $\mathrm{Wu}$ et al. explain the same relationship using the frequency of secondary corrective saccades [60]. To determine whether the differences between selection time and first saccade end times displayed in Fig. 1 can be explained by secondary and potentially tertiary saccades, we calculated the average number of saccades between target onset and successful selection. This analysis included only trials in which a selection was successfully achieved ("hits").

The average number of saccades is displayed in Fig. 4. The top sub-figure summarizes the number of saccades as a function of target size, the bottom as a function of ID (same values as Fig. 1). When reported by target size (averaged across saccade amplitudes), values ranged between one and two saccades (1.07-1.86). The same was true when plotted as a function of ID (1.05-1.87). Similar to [60], there was a higher likelihood of more than one saccade for targets with higher ID values. This suggests that only a single saccade was used to reach the target most of the time when below the critical ID, leading to a ballistic movement time profile rather than one that approximates Fitts' Law.

\section{Selection Performance}

Interaction user studies often report error rates, usually as frequency or ratio of trials in which a successful selection was not made due to user mistakes or technical errors (e.g., timeout). However, often the main argument is still based on movement time or throughput. This is appropriate for a highly accurate and precise input device like a mouse, where positional accuracy is on the pixel level. Current eye tracking systems on the other hand typically reach accuracies between 0.5 and 5 degrees, which means that detected gaze positions will be outside of the target at least some of the time, even when participants make highly accurate eye movements. For this reason, we applied a method inspired by psychophysics 

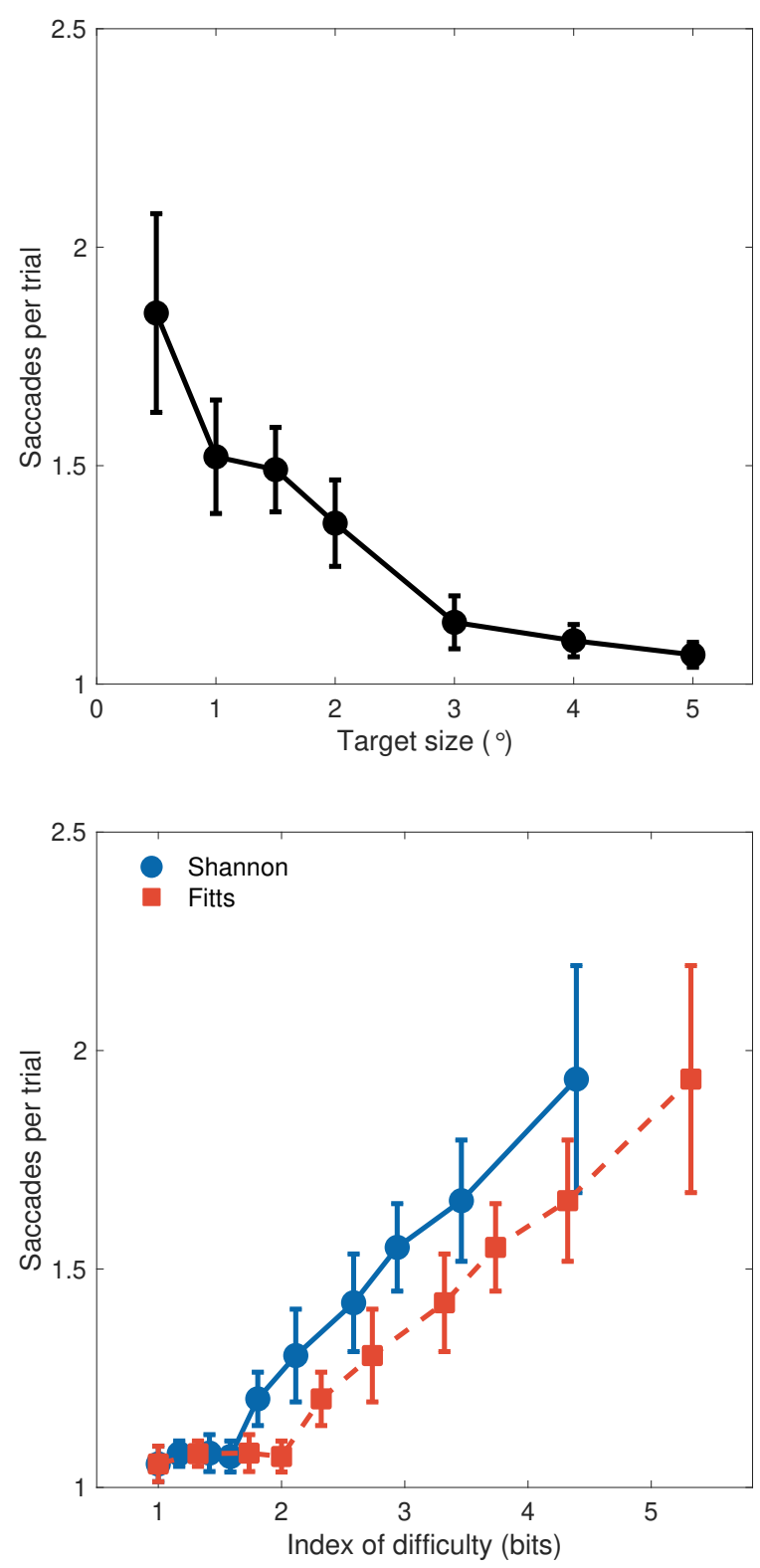

Figure 4: Average number of saccades per trial, measured between target onset and successful selection of the target (unsuccessful trials removed). Top: Number of saccades by target size, averaged across movement distances. Bottom: Number of saccades as a function of ID, computed using the Fitts (red squares) and Shannon (blue circles) formulation. Error bars indicate $\pm 1 \mathrm{SE}$.

and report selection probability (or "hit rate") as a function of target size, since this is the variable most affected by low spatial tracking accuracy.

Selection probabilities were determined by computing the saccade endpoint or median position during the sampling

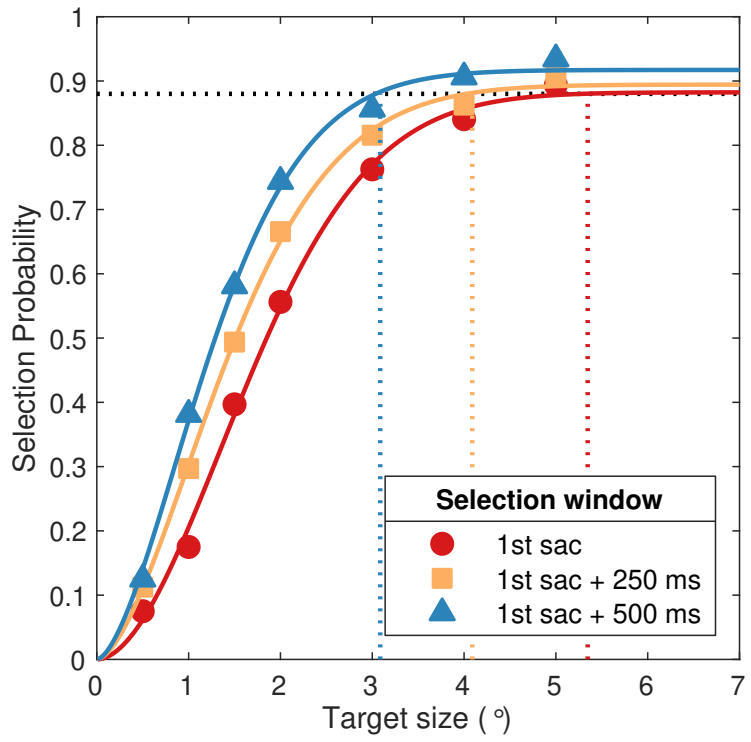

Figure 5: Selection performance by target size and sampling window size. Solid circles indicate measured selection performance (hit rate) for corresponding target sizes, solid lines indicate fitted Weibull CDFs. Dotted vertical lines indicate $\mathbf{8 8 \%}$ selection performance thresholds.

window and detecting whether the resulting position lay within the target circle. Distances equal to the target radius or smaller were scored as a "hit", all others as a "miss" (this includes trials with missing gaze positions due to blinks or other artifacts). Hit rates were then computed by aggregating the number of hits for each target size, first across all trials for each participant, then across participants. This analysis was repeated for all possible sampling window sizes from $0 \mathrm{~ms}$ (first saccade offset only) to $1500 \mathrm{~ms}$ after the offset of the first saccade. Selection probability for three exemplary window sizes $(0,250$ and $500 \mathrm{~ms})$ is plotted by target size as individual data points in Fig. 5. Hit rates for the first saccade only ranged from 0.08 ( $0.5^{\circ}$ target, average eye tracker accuracy) to 0.89 ( $5^{\circ}$ target) and this range increased to $0.13-0.94$ when a sampling window of $500 \mathrm{~ms}$ after the first saccade was used.

To determine target size thresholds for a given performance level, we next fit a Weibull cumulative distribution function to the resulting performance curves using nonlinear regression, with an added scaling parameter to capture the error rate in each case. Solid lines in Fig. 5 indicate the resulting functional relationship between target size and performance. Curves are shifted left with longer sampling windows, suggesting that averaging across more gaze samples can achieve the same level of selection performance for smaller target sizes. 


\section{Secondary Saccades Predict Optimal Dwell Times}

If capturing secondary saccades increases gaze accuracy with respect to the target, using longer sampling windows should lead to lower selection thresholds. In other words, this would allow the same level of selection performance with smaller targets. To test this assumption, we calculated the target sizes corresponding to $80 \%$ and $68 \%$ performance thresholds from the Weibull fit and compared them across window sizes. Additionally, we did the same for a threshold of $88 \%$, which was the highest threshold value that was reliably estimated for all window sizes. The dotted vertical lines in Fig. 5 indicate threshold target size for the chosen example windows, which decreased with increasing window size $(88 \%$ threshold at times in Fig. 5: $5.35^{\circ}, 4.09^{\circ}, 3.09^{\circ}$ ).

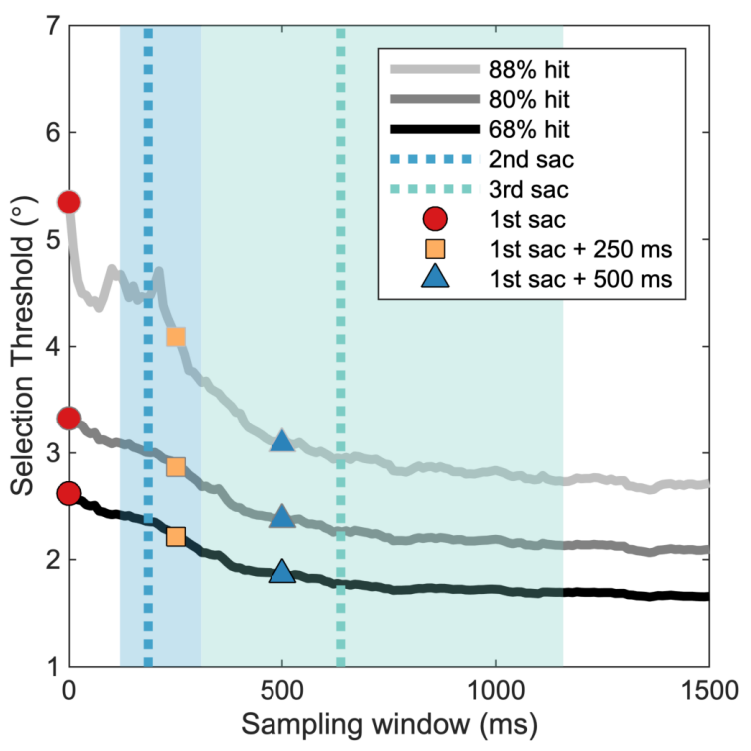

Figure 6: Target size thresholds (i.e., minimum target size for given performance level) for $68 \%, 80 \%$ and $88 \%$ selection performance, plotted as a function of sampling window size. Vertical lines indicate median saccade offset latencies for the second and third saccades (i.e., time after the end of the first saccade), shaded areas represent second and third quantiles.

To better visualize the change in threshold size with increasing window size, Fig. 6 plots the $88 \%, 80 \%$ and $68 \%$ performance thresholds as a function of sampling window size. Vertical dotted lines display the median end times of the second and third saccades, and all time series are aligned with the end time of the first saccade (i.e., they represent possible "dwell times"). Shaded bars indicate second and third quantiles (inner 50\%) for second and third saccade end times. The largest drop in threshold occurred around the median second saccade end time (183 ms after the first saccade), and all thresholds plateau at about $500 \mathrm{~ms}$. In light of these findings, we propose the third quantile of second saccade end times (capturing 75\% of corrective saccades) as a good dwell time measure (here: $309 \mathrm{~ms}$ ), which is in good agreement with dwell times reported across the literature (200 ms [43] $700 \mathrm{~ms}$ [61]). Choosing a different quantile would allow for adjustment of this threshold at the cost of a longer dwell time.

\section{DISCUSSION}

In the present study, we show that a relationship between target difficulty and movement time akin to Fitts' Law can emerge in gaze selection paradigms despite the ballistic nature of individual saccades. This phenomenon is rooted in human corrective saccades, performed mostly towards small targets, and can be a valuable tool to evaluate the performance of gaze-based interactions, as long as these specific oculomotor properties are kept in mind. We found that a linear relationship with ID predicted selection time well for targets above the $I D_{\text {crit }}$ (i.e., 1.42 for the Fitts and 1.74 for the Shannon formula, corresponding to $>3^{\circ}$ targets). The differences in movement time corresponded to an increasing frequency of corrective saccades for smaller targets. Below the critical ID value, selection times were approximately constant, which coincided with targets that could be selected using a single saccade in almost all cases. As expected due to the ballistic properties of saccades, end times of the first saccade in each trial were approximately constant and never predicted by ID. Besides the movement time analyses, targets that could be selected with a single saccade typically had high selection performance (around 90\%, equaling $10 \%$ error rate), but selection performance was limited by eye tracker fidelity and fell off rapidly for smaller target sizes. Selection performance for all target sizes improved when eye movements were sampled for at least $250 \mathrm{~ms}$ to capture secondary corrective saccades, and this increase in performance leveled off after around $500 \mathrm{~ms}$. These findings can serve as guidelines to create robust and performant gaze interaction designs in the future.

\section{Application of Fitts' Law to Eye Gaze}

Fitts' Law as a tool to evaluate gaze interaction methods has been extensively investigated in the past three decades, but mostly with a focus on comparing "eye pointing" to classical interaction methods such as keyboard and mouse $[22,43,56,61]$. At the same time, oculomotor literature has provided insights into the fundamental differences between gaze control and limb pointing movements [1, 26, 33, 60]. Our focus here was to bridge the gap between fields and make concrete recommendations where Fitts-like prediction of selection times could be useful, while detailing the differences between eye and hand motor control strategies as well as highlighting the limitations of Fitts' Law to the modeling of eye movement dynamics. 
Participants made one to two saccades per trial on average, with a higher probability of secondary saccades for targets that were smaller and/or had large IDs. This is in agreement with the findings of [60] on saccadic sequences, as well as theoretical work that explains a Fitts-like dependence of movement time on ID with as few as two submovements, one main and potentially one additional movement [26]. The finding that the number of saccades depends on target difficulty has direct implications for interaction design. For example, this means that targets that can be reached using a single saccade achieve practically constant selection times and low error rates. In our case, this was true for ID $<1.4$ or 1.7 , respectively, and target sizes above $3^{\circ}$ (selection times for targets of $4-5^{\circ}$ were in fact virtually identical to first saccade end times, cf. Fig. 3), but these numbers are likely to depend on the accuracy, precision, and detection algorithms of the eye tracking system used and these parameters should always be evaluated for a given system. Interaction schemes that rely on saccade detection instead of raw eye position already exist (e.g., [27, 31]) and have the potential to yield much faster selection times than those based on dwell time. Similar specifications would apply for passive selection tasks such as predicting user intent from fixated targets [5]. In any case, implementations should ensure that target selection in this ballistic range of difficulty are evaluated not using Fitts' Law, but using an appropriate model such as Carpenter's formula for movement time [9].

For targets above the critical ID value of 1.4/1.7, both Fitts' Law formulations predicted movement times in a large part due to the increased frequency of secondary saccades. Secondary saccade rates increased for small targets, in good agreement with oculomotor literature [33, 60], and allowing for enough gaze samples to capture these secondary saccades increased selection performance. This is well in line with the established practice of adding a dwell time of between 200 and $750 \mathrm{~ms}$ after gaze enters a target and confirms that the successful application of dwell time is grounded in corrective saccade behavior. Notably, the only classical gaze interaction study that reported a better fit for a ballistic model than Fitts' Law used a very short dwell time of only $150 \mathrm{~ms}$ [51]. Recent work presented at ETRA reported a new technique to reduce dwell time to $80-100 \mathrm{~ms}$ [27]. While this number seems much lower than other reported values, their algorithm uses knowledge about the preceding saccade to determine whether it was on target and correctly predicted by Fitts' Law before starting the dwell time counter.

\section{Limitations and Future Work}

Fitts' original publications specify his work as a way to quantify the information capacity of the human motor system [16], but later work leading up to the ISO 9241-9 standard applied the same formulation as a method to characterize and compare interactive systems $[17,61]$. When it comes to gaze as an input method, any performance metric necessarily captures a combination of human behavior and the accuracy of the corresponding eye tracking device, and thus our results are most applicable for our tracker at $0.5^{\circ}$ average accuracy. Unsurprisingly, studies reporting much lower eye tracking accuracies found gaze to be a poorer selection method than head orientation or the mouse $[22,46]$.

There is an ongoing discussion in the research community around the "correct" formulation of ID (see. e.g. [13, $24,36,38]$ ), related to the model's origins in information theory and how well different formulations of ID can capture experimental data [13]. Given the strong differences in theoretical background between Fitts' Law and oculomotor control, our goal here was not to recommend a formulation to apply to eye gaze selection times. Rather, we aim to highlight that a Fitts-like pattern of movement times can nevertheless emerge from a motor control process that is very different from Fitts' original work on arm and hand movements. For comparison with existing work, we decided to report model parameters for two formulations: Fitts' original formula for ID [16, 24] and the Shannon formulation suggested by MacKenzie [36, 38]. Fitts' variant has been reported as the "most valid form based on information theory" [24], while many recent studies on gaze interaction report results using the Shannon formulation (e.g., [21, 27, 46, 61]), likely due to its inclusion in the ISO 9421-9 standard [17]. As shown in Fig. 1 and Table 2, both models yielded a comparable model fit on our data.

More recent work on using Fitts' Law for evaluation of pointing tasks, including the ISO standard [17], suggest to fit the model not using theoretical IDs derived from the experimental conditions but from the variability of movement endpoints produced by the participants, thus capturing their actual performance $[37,57]$. We opted not to perform this correction here because gaze positions used to determine selection were calculated using different algorithms for selection times (first sample of a moving window), saccade end times (velocity- and acceleration based algorithm), and selection windows (median of multiple samples). We have no reason to assume that the variabilities of thus determined endpoints would follow similar patterns and therefore report models for the theoretical values of ID instead.

A recent paper pointed out that specific combinations of distance and target size values lead to strong correlations between either target distance or size and the resulting IDs [19]. Such correlations could then artificially inflate the model fit $\left(R^{2}\right)$, together with the frequently employed strategy of averaging over conditions with different distances and target sizes but identical ID. The authors cite Miniotas' [43] results on eye gaze as an example, arguing that a strong confound of movement distance with ID inflated the model fit for Fitts' 
Law, while a competing model such as the saccade duration formula of Carpenter [9] could have explained the data equally well. While our chosen combinations of two movement amplitudes and seven target sizes do not fall into the critical range of values reported by Gori et al. [19], our above analysis does average over identical IDs. However, when reanalyzed by fitting the regression line to the full range of tested conditions, the pattern shown in Fig. 1 did not change qualitatively: selection time (independent of individual saccades) still followed a Fitts' Law-like pattern (Shannon ID: a $=44.4 \mathrm{~ms}, \mathrm{~b}=157.6 \mathrm{~ms} / \mathrm{bit}, R^{2}=.86$ ), while there was still no influence of first saccade offset latencies on movement time $\left(\mathrm{a}=267.8 \mathrm{~ms}, \mathrm{~b}=4.8 \mathrm{~ms} / \mathrm{bit}, R^{2}=.10\right)$. Because we used only two saccade amplitudes $\left(5\right.$ and $\left.10^{\circ}\right)$, our data do not allow for a full model comparison between Fitts' Law and Carpenter's formula. Along similar lines, the range of amplitudes tested here is likely too small to show a reliable effect of saccade amplitude on movement time as predicted by the main sequence $[1,9,23,58]$. Future user studies should explore a broader range of saccade amplitudes to explore whether MT as predicted by Carpenter's formula [9] significantly contributes to gaze selection times at larger amplitudes. Besides movement time predicted by the main sequence, a complete model of gaze selection times and accuracy would need to encompass endpoint inaccuracies resulting from saccadic gain [33], frequency and amplitude of secondary saccades [60], as well as the accuracy and precision of the specific eye tracker used. While beyond the scope of this manuscript, such a full model of gaze interaction would certainly be desirable as a basis for UX design and is an obvious candidate for further research.

Previous work has also raised the question of what exactly constitutes a target in a gaze selection task [13]. In the case of explicit targets such as geometric shapes or icons of different size, this is reduced to the question of a specific landing position within the target area. Oculomotor research found that saccades to spatially extended targets are generally aimed to the center of gravity of the target shape with considerable accuracy $[33,44]$. While this applies to the circular targets used here, our results can not inform about implicit visual targets that need to be extracted from a complex visual scene, for example using bottom-up visual saliency [28].

\section{CONCLUSION}

Our results tie together existing work on Fitts' Law for eye gaze with findings from the oculomotor and motor control literature. The Fitts-like model of selection time described here can be a highly useful metric for the evaluation of gazebased selection paradigms, but it comes with caveats: such paradigms should allow for enough time to capture not only the primary saccade towards the target but also any secondary corrective eye movements, likely to occur for smaller targets with greater Index of Difficulty. Based on our findings, we offer the following concrete recommendations to anyone looking to design a gaze-based interaction system:

(1) Leverage the ballistic nature of eye movements. If design allows, plan for target sizes and ID values in the ballistic region (ID < 1.4) to achieve near-constant selection times. Such a paradigm could potentially utilize detected saccade events directly, greatly increasing interaction speed and robustness.

(2) Know when to apply ID models. When using Fitts' Law to compare gaze to other input methods, ensure that specified ID values are above the corresponding $I D_{\text {crit }}$ and that enough data is captured to include corrective eye movements.

(3) Closely evaluate your eye tracking system. Low eye tracking fidelity is not necessarily detectable from analyses of movement times or Fitts' Law parameters, but strongly influences selection performance for smaller target sizes, potentially making for a frustrating experience.

\section{ACKNOWLEDGMENTS}

We thank Hrvoje Benko for helpful discussions and Mark Opaliski for help with data collection.

\section{REFERENCES}

[1] A Terry Bahill, Michael R Clark, and Lawrence Stark. 1975. The main sequence, a tool for studying human eye movements. Mathematical Biosciences 24, 3-4 (1975), 191-204.

[2] Michael Barz, Florian Daiber, Daniel Sonntag, and Andreas Bulling. 2018. Error-Aware Gaze-Based Interfaces for Robust Mobile Gaze Interaction. (2018).

[3] W Becker. 1972. The control of eye movements in the saccadic system. Bibliotheca ophthalmologica: supplementa ad ophthalmologica 82 (1972), 233-243.

[4] W Becker and AF Fuchs. 1969. Further properties of the human saccadic system: eye movements and correction saccades with and without visual fixation points. Vision research 9, 10 (1969), 1247-1258.

[5] Roman Bednarik, Hana Vrzakova, and Michal Hradis. 2012. What do you want to do next: a novel approach for intent prediction in gazebased interaction. In Proceedings of the symposium on eye tracking research and applications. ACM, 83-90.

[6] Jonas Blattgerste, Patrick Renner, and Thies Pfeiffer. 2018. Advantages of eye-gaze over head-gaze-based selection in virtual and augmented reality under varying field of views. In Proceedings of the Workshop on Communication by Gaze Interaction. ACM, 1.

[7] Bruce Bridgeman, Derek Hendry, and Lawrence Stark. 1975. Failure to detect displacement of the visual world during saccadic eye movements. Vision research 15, 6 (1975), 719-722.

[8] David C Burr, M Concetta Morrone, and John Ross. 1994. Selective suppression of the magnocellular visual pathway during saccadic eye movements. Nature 371, 6497 (1994), 511.

[9] Roger HS Carpenter. 1988. Movements of the Eyes, 2nd Rev. Pion Limited.

[10] Haiyin Chen-Harris, Wilsaan M Joiner, Vincent Ethier, David S Zee, and Reza Shadmehr. 2008. Adaptive control of saccades via internal feedback. Journal of Neuroscience 28, 11 (2008), 2804-2813. 
[11] ERFW Crossman and PJ Goodeve. 1983. Feedback control of handmovement and Fitts' law. The Quarterly fournal of Experimental Psychology Section A 35, 2 (1983), 251-278.

[12] Heiko Drewes. 2010. Eye gaze tracking for human computer interaction. Ph.D. Dissertation. lmu.

[13] Heiko Drewes. 2010. Only one Fitts' law formula please!. In CHI'10 Extended Abstracts on Human Factors in Computing Systems. ACM, 2813-2822.

[14] Anna Maria Feit, Shane Williams, Arturo Toledo, Ann Paradiso, Harish Kulkarni, Shaun Kane, and Meredith Ringel Morris. 2017. Toward everyday gaze input: Accuracy and precision of eye tracking and implications for design. In Proceedings of the 2017 CHI Conference on Human Factors in Computing Systems. ACM, 1118-1130.

[15] David J Field. 1987. Relations between the statistics of natural images and the response properties of cortical cells. fosa a 4, 12 (1987), 23792394.

[16] Paul M Fitts. 1954. The information capacity of the human motor system in controlling the amplitude of movement. fournal of experimental psychology 47, 6 (1954), 381.

[17] International Organization for Standardization. 2000. Ergonomic Requirements for Office Work with Visual Display Terminals (VDTs): Requirements for non-keyboard input devices. ISO.

[18] Khai-Chung Gan and Errol R Hoffmann. 1988. Geometrical conditions for ballistic and visually controlled movements. Ergonomics 31, 5 (1988), 829-839.

[19] Julien Gori, Olivier Rioul, Yves Guiard, and Michel Beaudouin-Lafon. 2018. The Perils of Confounding Factors: How Fitts' Law Experiments can Lead to False Conclusions. In Proceedings of the 2018 CHI Conference on Human Factors in Computing Systems. ACM, 196.

[20] Sven-Thomas Graupner, Michael Heubner, Sebastian Pannasch, and Boris M Velichkovsky. 2008. Evaluating requirements for gaze-based interaction in a see-through head mounted display. In Proceedings of the 2008 symposium on Eye tracking research \& applications. ACM, 91-94.

[21] John Paulin Hansen, Anders Sewerin Johansen, Dan Witzner Hansen, Kenji Itoh, and Satoru Mashino. 2003. Command without a click: Dwell time typing by mouse and gaze selections. In Proceedings of Human-Computer Interaction-INTERACT. 121-128.

[22] John Paulin Hansen, Vijay Rajanna, I Scott MacKenzie, and Per Bækgaard. 2018. A Fitts' law study of click and dwell interaction by gaze, head and mouse with a head-mounted display. In Proceedings of the Workshop on Communication by Gaze Interaction. ACM, 7.

[23] Christopher M Harris and Daniel M Wolpert. 2006. The main sequence of saccades optimizes speed-accuracy trade-off. Biological cybernetics 95, 1 (2006), 21-29.

[24] Errol R Hoffmann. 2013. Which version/variation of Fitts' law? A critique of information-theory models. fournal of motor behavior 45, 3 (2013), 205-215.

[25] Errol R Hoffmann. 2016. Critical index of difficulty for different body motions: A review. Journal of motor behavior 48, 3 (2016), 277-288.

[26] Errol R Hoffmann. 2016. Fitts' Law With An Average of Two or Less Submoves? Journal of motor behavior 48, 4 (2016), 318-331.

[27] Toshiya Isomoto, Toshiyuki Ando, Buntarou Shizuki, and Shin Takahashi. 2018. Dwell time reduction technique using Fitts' law for gazebased target acquisition. In Proceedings of the 2018 ACM Symposium on Eye Tracking Research \& Applications. ACM, 26.

[28] Laurent Itti, Christof Koch, and Ernst Niebur. 1998. A model of saliencybased visual attention for rapid scene analysis. IEEE Transactions on pattern analysis and machine intelligence 20, 11 (1998), 1254-1259.

[29] Robert JK Jacob. 1990. What you look at is what you get: eye movementbased interaction techniques. In Proceedings of the SIGCHI conference on Human factors in computing systems. ACM, 11-18.

[30] Richard J Jagacinski and Donald L Monk. 1985. Fitts' Law in Two dimensions with hand and head movements movements. fournal of motor behavior 17, 1 (1985), 77-95.

[31] Oleg V Komogortsev, Young Sam Ryu, Do Hyong Koh, and Sandeep M Gowda. 2009. Instantaneous saccade driven eye gaze interaction. In Proceedings of the International Conference on Advances in Computer Enterntainment Technology. ACM, 140-147.

[32] Eileen Kowler. 2011. Eye movements: The past 25years. Vision research 51, 13 (2011), 1457-1483.

[33] Eileen Kowler and Erik Blaser. 1995. The accuracy and precision of saccades to small and large targets. Vision research 35, 12 (1995), 1741-1754.

[34] Jui-Feng Lin and Colin G Drury. 2010. Modeling Fitts' law. In The 9th Pan-Pacific Conference on Ergonomics.

[35] Ray F Lin and Yi-Chien Tsai. 2015. The use of ballistic movement as an additional method to assess performance of computer mice. International fournal of Industrial Ergonomics 45 (2015), 71-81.

[36] I Scott MacKenzie. 1989. A note on the information-theoretic basis for Fitts' law. Journal of motor behavior 21, 3 (1989), 323-330.

[37] I Scott MacKenzie. 1992. Fitts' law as a research and design tool in human-computer interaction. Human-computer interaction 7, 1 (1992), 91-139.

[38] Ian Scott MacKenzie. 2013. A note on the validity of the Shannon formulation for Fitts' index of difficulty. Open fournal of Applied Sciences 3, 06 (2013), 360.

[39] Päivi Majaranta. 2011. Gaze Interaction and Applications of Eye Tracking: Advances in Assistive Technologies: Advances in Assistive Technologies. IGI Global.

[40] Päivi Majaranta and Andreas Bulling. 2014. Eye tracking and eye-based human-computer interaction. In Advances in physiological computing. Springer, 39-65.

[41] Päivi Majaranta, I Scott MacKenzie, Anne Aula, and Kari-Jouko Räihä. 2006. Effects of feedback and dwell time on eye typing speed and accuracy. Universal Access in the Information Society 5, 2 (2006), 199208.

[42] David E Meyer, Richard A Abrams, Sylvan Kornblum, Charles E Wright, and JE Keith Smith. 1988. Optimality in human motor performance: ideal control of rapid aimed movements. Psychological review 95, 3 (1988), 340.

[43] Darius Miniotas. 2000. Application of Fitts' law to eye gaze interaction. In CHI'O0 extended abstracts on human factors in computing systems. ACM, 339-340.

[44] Christoph J Ploner, Florian Ostendorf, and Sandra Dick. 2004. Target size modulates saccadic eye movements in humans. Behavioral neuroscience 118, 1 (2004), 237.

[45] C Prablanc, D Masse, and JF Echallier. 1978. Error-correcting mechanisms in large saccades. Vision research 18, 5 (1978), 557-560.

[46] Yuan Yuan Qian and Robert J. Teather. 2017. The Eyes Don't Have It: An Empirical Comparison of Head-based and Eye-based Selection in Virtual Reality. In Proceedings of the 5th Symposium on Spatial User Interaction (SUI '17). ACM, New York, NY, USA, 91-98. https://doi. org/10.1145/3131277.3132182

[47] Vijay Rajanna and John Paulin Hansen. 2018. Gaze typing in virtual reality: impact of keyboard design, selection method, and motion. In Proceedings of the Tenth Biennial ACM Symposium on Eye Tracking Research and Applications (ETRA'18).

[48] John Ross, M Concetta Morrone, Michael E Goldberg, and David C Burr. 2001. Changes in visual perception at the time of saccades. Trends in neurosciences 24, 2 (2001), 113-121.

[49] Javier San Agustin, Henrik Skovsgaard, John Paulin Hansen, and Dan Witzner Hansen. 2009. Low-cost Gaze Interaction: Ready to 
Deliver the Promises. In $\mathrm{CHI}$ '09 Extended Abstracts on Human Factors in Computing Systems (CHI EA '09). ACM, New York, NY, USA, 4453-4458. https://doi.org/10.1145/1520340.1520682

[50] Stephen H Scott. 2004. Optimal feedback control and the neural basis of volitional motor control. Nature Reviews Neuroscience 5, 7 (2004), 532.

[51] Linda E Sibert and Robert JK Jacob. 2000. Evaluation of eye gaze interaction. In Proceedings of the SIGCHI conference on Human Factors in Computing Systems. ACM, 281-288.

[52] Linda E Sibert, James N Templeman, and Robert J Jacob. 2001. Evaluation and analysis of eye gaze interaction. Technical Report. NAVAL RESEARCH LAB WASHINGTON DC.

[53] Vildan Tanriverdi and Robert JK Jacob. 2000. Interacting with eye movements in virtual environments. In Proceedings of the SIGCHI conference on Human Factors in Computing Systems. ACM, 265-272.

[54] Emanuel Todorov and Michael I Jordan. 2002. Optimal feedback control as a theory of motor coordination. Nature neuroscience 5, 11 (2002), 1226 .
[55] AJ Van Opstal and JAM Van Gisbergen. 1989. Scatter in the metrics of saccades and properties of the collicular motor map. Vision research 29, 9 (1989), 1183-1196.

[56] Colin Ware and Harutune H Mikaelian. 1987. An evaluation of an eye tracker as a device for computer input. In Acm sigchi bulletin, Vol. 17. ACM, 183-188.

[57] Alan Traviss Welford. 1968. Fundamentals of skill. (1968).

[58] Gerald Westheimer. 1954. Mechanism of saccadic eye movements. AMA Archives of Ophthalmology 52, 5 (1954), 710-724.

[59] Alan M Wing. 1983. Crossman and Goodeve (1963): Twenty years on. The Quarterly fournal of Experimental Psychology Section A 35, 2 (1983), 245-249.

[60] Chia-Chien Wu, Oh-Sang Kwon, and Eileen Kowler. 2010. Fitts's Law and speed/accuracy trade-offs during sequences of saccades: Implications for strategies of saccadic planning. Vision Research 50, 21 (2010), $2142-2157$.

[61] Xuan Zhang and I Scott MacKenzie. 2007. Evaluating eye tracking with ISO 9241-part 9. In International Conference on Human-Computer Interaction. Springer, $779-788$. 